\title{
Overview of Research Challenges towards Smart Grid Quality by Design
}

\author{
David Gešvindr \\ Masaryk University \\ Faculty of Informatics \\ Brno, Czech Republic \\ Email: gesvindr@mail.muni.cz
}

\author{
Barbora Buhnova \\ Masaryk University \\ Faculty of Informatics \\ Brno, Czech Republic
}

Email: buhnova@mail.muni.cz

\author{
Jan Rosecky \\ Masaryk University \\ Faculty of Informatics \\ Brno, Czech Republic \\ Email: j.rosecky@mail.muni.cz
}

\begin{abstract}
Power distribution systems worldwide are entering one of the biggest transformations since their inception. The Smart Grid has become a strategic term, which is associated with promising technology enhancement on one hand, but also high investment risks on the other hand. To maximize the former and minimize the latter, power distribution companies are searching for methods and tools to simulate and compare different Smart Grid design alternatives and deployment scenarios.

In this paper, we draw upon our involvement in the modelling and simulation of the Smart Grid in the Czech Republic, and elaborate possible directions of Smart Grid modelling and analysis research to reach the "quality by design" before the actual Smart Grid realization. The discussion then details a specific design challenge together with its context within the design of the Czech Smart Grid.
\end{abstract}

\section{INTRODUCTION}

$\mathbf{T}$ HE CURRENT generation of power distribution networks faces new requirements that stimulate discussion on its future transformation. Some of the strongest triggers that the current power grid fails to keep pace with are the transition to a distributed power generation [1] and demand response optimization [2], whose both rely on mature information exchange and control. This stimulated the integration of the power distribution and information technology domains into the concept of a Smart Grid. The Smart Grid is an electricity network that can cost-efficiently integrate the behavior and actions of all users connected to it-generators, consumers and those that do both-in order to ensure economically efficient, sustainable power system with low losses and high levels of quality and security of supply [3]. The Smart Grid relies on an Advanced Metering Infrastructure (AMI) [4], [2] with a bidirectional communication channel which allows the control of electricity demand at customer level by directly or indirectly (e.g. by on-line adjusting electricity prices) controlling household appliances [2].

The deployment of Smart Grids is becoming a strategic act for many countries, forced by their legislation [5]. European Commission calls in its mandate M411 [6], released in 2009, for the standardization in the area of Smart Metering. The standards should enable interoperability of utility meters (water, gas, electricity, heat), and allow mass production of Smart Meter devices at the competitive European market. The mandate is aligned with the OPEN meter project [7], which ended in June 2011 and delivered open and public standards for smart metering, and consequent OpenNode project [8] with similar endevour regarding low-voltage monitoring.

The transition to the Smart Grid represents heavy investments (in orders of hundreds of millions of Euros) into technology that was never before deployed in such a large scale. To minimize investment risks and maximize benefits, power distribution companies are seeking new ways of Smart Grid modelling and simulation to evaluate and compare different Smart Grid architectures and deployment scenarios. This initiatives aim in each national context at a cost- and quality-effective validated solution, before mass investments into Smart Grid deployment.

Goal of the paper: In this paper, we draw upon our involvement in the modelling and simulation of the envisioned Smart Grid in the Czech Republic, and elaborate a discussion on the possible directions of Smart Grid modelling and analysis to reach the "quality by design" before the actual Smart Grid deployment.

Paper structure: The paper is organized as follows: Section II overviews the history, context and key terminology of the field; Section III discusses the specific research questions related to the modelling, simulation and analysis towards Smart Grid design; Section IV elaborates a specific research question concerned with the communication strategy discussed in the specific context of the Czech Smart Grid design; and Section V concludes the paper.

Related work: Although numerous overview papers have been published in the Smart Grid domain, the discussion of research questions and challenges towards mature Smart Grid design is not receiving systematic attention. The existing surveys instead focus on the goals, impact, benefits, risks and undelaying technology principles (architecture, communication technologies) of the Smart Grid, usually influenced by national research and deployment [4], [9], [2], [10]. The source of the information are hence the research papers elaborating individual research questions in isolation, such as for instance the papers on multi-agent approaches in a distributed Smart Grid [11], [12], [13], security-driven Smart Grid design [14], [15], [16], survivability analysis and Smart Grid self-repair [11], [17], or demand response optimization [18]. 


\section{BACKGROUND}

One of the first large network utilities was built by Thomas Alva Edison in 1882 in New York. Edison promoted direct current for electric power distribution, despite the disadvantages of this approach, namely in short distance between a power plant and its customers $(2.4 \mathrm{~km}$ was the limit of effective distance), which meant that small power plants needed to be built in customer areas. New research in Europe and America has brought electric motors and transformers working with alternating current and with the help of Edisons major opponent George Westinghouse, the previously used direct current was replaced with the alternating current, which persisted till today. Alternating current could be efficiently transmitted over long distance and hence allowed power plants to be built outside inhabited areas, closer to input resources.

The process of power transmission nowadays involves stepup transformers that transform electricity into very high voltage to decrease transmission losses. Electricity is then transmitted over hundreds of kilometers of transmission network. Before distribution to end customers, the voltage is decreased by a series of step-down transformers, which act as gates between high voltage power transmission and low voltage power distribution networks.

In the Czech Republic the power transmission network consists of power plants, first-level substations and highvoltage power lines. The first-level substations and power plants are connected using $400 \mathrm{kV}$ and $220 \mathrm{kV}$ lines. There is high flow of energy and therefore very high voltage is used to lower the electric current, which leads to lower losses during transmission. The first-level substations produce also $110 \mathrm{kV}$ intended for power distributors.

The Czech power distribution network consists of $110 \mathrm{kV}$ lines, second-level substations transforming $110 \mathrm{kV}$ to $22 \mathrm{kV}$ or $35 \mathrm{kV}$ for connected third-level substations. Multiple thirdlevel substations are situated in towns and produce well-known $230 \mathrm{~V} / 400 \mathrm{~V}$ used by end customers.

The current generation of power transmission and distribution network has been designed for centralized electricity generation in a small number of large power plants. Nowadays, this concept is being replaced with a more distributed electricity generation thanks to an increasing amount of predominantly renewable energy sources. European Union supports renewable energy sources in its Energy and Climate Change Policy [20] with the goal to achieve $20 \%$ renewables share in the European energy mix until 2020.

The new approach, however, brings up serious issues: (1) Since the grid load changes along the day and since there is no efficient way to centrally store the surplus of generated electricity for later use or to rapidly increase the demand of electricity, power plants have to adjust their electricity production to meet the expected demand. Immediate production change is very complicated for most types of power plants, so both the consumption and the production of the renewables have to be predicted in advance, which brings additional risks to the whole process of electricity production and distribution. Moreover, thoughtless connection of many uncontrolled renewables (e.g. photovoltaic panels) may (2) damage the current grid or (3) cause significant technical losses, because the amount of generated electricity may in specific areas exceed its demand and overload the related part of the grid.

The first step towards effective management of power distribution is Smart Metering, which measures and collects power consumptions of individual customers. In particular, the deployment of Smart Metering can provide the following inputs for smart power distribution management:

a) Collection of power consumption data: Without deployment of Smart Meter units, the power consumption measurements are automatically collected only during electricity transmission in large substations. In the Czech Republic, moreover, only very large customers have similar devices already deployed. Collection of more data from individual consumers and all substations is a necessary condition of reliable predictions, which can help to locally balance the grid load.

b) Early fault detection: Current generation of power grids can only detect failures in substations that are connected via computer network to a control center that monitors the grid. It is also possible to detect broken high-voltage wires. But when it comes to individual customers, it does not provide means to detect power outages at individual customer level, because such an outage cannot be detected directly from the substation. In the Czech Republic, this type of outage is usually reported by the customer via a phone call at distributors service line. Nowadays, to detect this type of outage automatically, each monitored customer needs to be equipped with a device that periodically sends hearth beat signals to the substation. Smart meter measurements sent to a substation can substitute the hearth beat signaling.

The current state of the practice in power transmission and distribution implies numerous challenges for the Smart Grid design and deployment, many of which could be adressed with multi-agent approaches. The next sesion discusses these challenges and related research questions in detail.

\section{SMART GRID MODELLING, SIMULATION AND ANALYSIS}

To minimize the risks associated with the Smart Grid deployment and maximize its quality across multiple quality attributes, possible modelling and simulation techniques to evaluate and compare different Smart Grid design alternatives before the actual deployment are becoming of high interest to power distribution companies. This section summarizes the specific research questions towards Smart Grid design that we identified with our industrial partners, and aims at opening the discussion on the newly establishing research field of Smart Grid design support, to which the computer science, and multiagent research in particular, can strongly contribute. The areas include: 


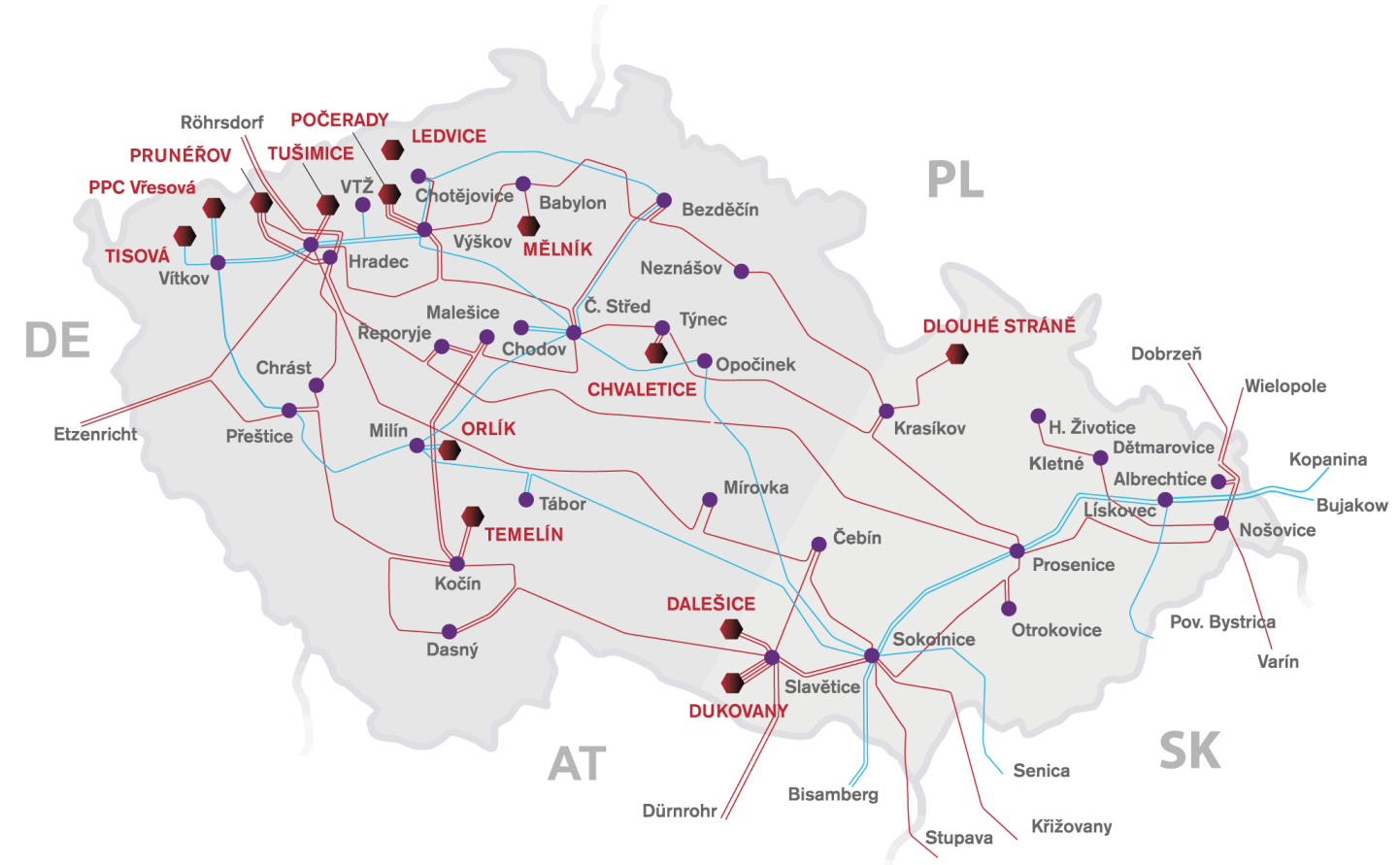

Fig. 1. Map of the Czech transmission network, where red labels are the major power plants, red lines are $400 \mathrm{kV}$ lines, purple dots are large substations and blue lines are $220 \mathrm{kV}$ lines [19].
A. Prediction of the expected consumption
B. Load control
C. Energy backup and storage
D. Hardware infrastructure design
E. Smart grid topology design
F. Decision on the types of information to be exchanged and transferred
G. Evaluation of Smart Grid communication strategies
H. Quality assessment of different Smart Grid design op- tions

\section{A. Prediction of the expected consumption}

Smart metering can significantly contribute to the creation of a new global power consumption model, recording power consumption even at an individual customer level. Such a model can be used for the prediction of expected electricity consumption. As mentioned in [2] a valid consumptionprediction model is much more complex than it used to be before, because of customer life style change. Also, a need to predict locally-dependent consumption and ability to react on lower electricity prices arises together with the requirement on local load-balancing.

We identified the following research questions associated with this topic:

- How to predict power consumption in both normal and crisis situations?

- What is the appropriate level of detail on which the consumption should be studied? How to cluster the Smart Grid architecture into consumption-relevant levels for analysis?
- What trends and technologies may significantly affect the validity of current prediction models? How to predict the consumption associated with the increasing use of electric automobiles?

\section{B. Load control}

The goal of the load control is to lower the gap between the base load and the daily peak load in each locality. This can be achieved by shifting power usage to off-peak hours, in particular to balance production of local renewables. There are multiple advantages of this approach: (1) It decreases the maximum power generation and transition capacity required to avoid blackouts; (2) It avoids extensive stopping and starting of power generating units by shaping the power usage to remain relatively constant over time [2]; (3) It helps avoiding electricity overflow from low voltage to high voltage and related problems mentioned above. This can be achieved either manually by motivating the customers to shift their power usage via variable pricing models or automatically by remote power distributor signaling that controls customer equipment.

In the Czech Republic, a combination of both mechanisms is used for households with water boilers which are controlled remotely via HDO signals ${ }^{1}$. The signals are broadcasted together with a code of the controlled area, so that only HDO switches in the targeted area are affected. Nevertheless, since these groups are fixed, one cannot smoothly control the loadbalance, hence this solution works only partially and cannot be considered sufficient for the uprising problems with local renewable energy sources.

${ }^{1} \mathrm{HDO}$ is a shortcut that stands for "Massive Remote Control" in Czech. 
There are algorithms that can optimize the power demand at the customer level based on the electricity price. One of them is described and validated in [18]. However, to validate such a demand response model in a global context, we need to stepup in the hierarchy and model many interconnected customers in the grid and mainly the behavior of the distributor, who sends impulses (in form of real-time price or direct control of appliances) to increase or decrease the demand.

The research questions that could be answered with the help of a complex demand response model are:

- Are the current communication channels suitable for reliable control of customer appliances or real-time delivery of the price? How does the system behave in case of a broken communication line?

- How effective is the proposed demand response model in terms of lowering the gap between the base load and the peak load? What is its response time?

- What is the overall reliability and safety of the proposed solution? What happens if the load control mechanism misbehaves? E.g. intelligent agents start to spread invalid information to other agents in the grid which leads towards a snowball effect? What protection needs to be implemented to ensure grid survivability in such a case?

\section{Energy backup and storage}

One of the consequences of high difference between the base and peak load in current power grids can be the need for large-scale (to balance the whole grid) and small-scale (to balance individual areas) energy storage. As for the large-scale energy storage, according to Electric Power Research Institute the pumped-storage hydroelectricity provides more than $99 \%$ of bulk storage capacity worldwide [21] with the efficiency that varies between $70 \%$ and $80 \%$.

Another approach to energy storage is the agent based micro-storage introduced by Vytelingum et al. [22]. This approach models the use of small capacity batteries $(4 \mathrm{kWh})$ deployed in households and accompanied with an intelligent agent, which may be an integral part of a Smart Meter. The agent buys electric energy when it is cheap and stores this energy in batteries for its use during peak time when energy price increases. According to the study, this system can achieve savings of up to $13 \%$ for an average customer using a 4 $\mathrm{kWh}$ battery. Moreover, in case of power outage, the system can work as an energy backup. Other approaches involve electromobile batteries or local hydrogen production.

The research questions associated with this domain are:

- How to predict required energy storage capacity in case of successful Smart Grid deployment?

- Shall households employ local small-capacity energy storage? Will their deployment affect the overall demandresponse strategy? Should they be strictly controlled by the utility provider or should they autonomously react on incentives (lower energy prices)?

- How will the demand response problem be affected by growing share of electro vehicles which are charged mostly over night or during working hours? Should be the chargers controlled remotely? How to balance the need of the user to charge the vehicle and the need of the grid to govern the demand? How to predict electric vehicle usage and its recharge power consumption?

- How to solve the problem of energy surplus when the demand response mechanism fails?

\section{Hardware infrastructure design}

The goal at least in the Czech Republic is to minimize hardware infrastructure investments and construction costs, and hence reuse as many existing infrastructure elements as possible when transiting to the Smart Grid. The suitability of every reused component should be individually assessed together with its parameters such as component dependencies, reliability, replacement costs and other associated limitations comparing to modern alternatives. Such an evaluation process, which is in fact multi-objective optimization, may be very slow and complex when done manually. Therefore, it is beneficial to employ a Smart Grid model with all relevant components modelled together with their dependencies and parameters. The model can be then used for semi-automatic or automatic evaluation of different versions of hardware components and their associated risks and benefits. The automation of this process may lead to more complex analysis of the model in a larger scale, which may identify previously hidden solutions that can be easily validated with running simulation on top of the grid models.

This approach is suitable for the modelling and validation of Smart Meter features, communication elements, and data concentrators. Even control and data-processing software can be evaluated in this way.

The most relevant research questions are:

- What design strategies can be used to maximize the quality of the Smart Grid under the constraint of legacy infrastructure?

- What hardware infrastructure changes will imply the highest ratio of quality increase to implementation costs (i.e. quality/cost)?

\section{E. Smart grid topology design}

The overall Smart Grid quality and behavior depends not only on the hardware components, but also on their organization into the grid topology. The topology, which determines the logical architecture of the grid, can take advantage of different levels of centralization (e.g. customer data from a given region managed through a single substation), hierarchical structure (e.g. substations governed by parent substations) and redundancy (to minimize the critical chain effects and maximize grid survivability in case of local outage).

The relevant research questions towards the logical architecture of a Smart Grid are the following:

- What shall be the level of centralization within the Smart Grid? 
- Shall the substations and other grid components be organized in a hierarchical manner? If yes, how many levels should be used?

- What level of homogeneity/heterogeneity (with respect to the smart logic within the grid nodes) is most beneficial?

- What are the critical chains of the Smart Grid with respect to the consequences of their outage?

- What is the level of grid robustness and survivability? What are the most cost-effective topology improvements that significantly increase robustness and survivability? What is the expected benefit of self-repair mechanisms comparing to their implementation costs?

\section{F. Decision on the types of information to be exchanged and transferred}

The concept of a Smart Grid relies on the transfer of various information between grid components. The Advanced Metering Infrastructure (AMI) needs to transfer measurement results collected from Smart Meters for processing purposes, substations need to report their state to a parent substation in case of hierarchical infrastructure or broadcast their state to other substations in case of multi-agent processing.

To minimalize the traffic among Smart Grid components, the information value of different types of data needs to be understood and used to drive the identification of needless data, whose transfer should be limited. On the other hand, the critical information types shall be prioritized in the communication.

The relevant research questions include:

- How to identify and model importance of collected measurements for later processing in the Smart Grid? How this process can be automated?

- What is the ideal collection frequency of measurements from Smart Meters to satisfy the legislation needs, ensure sufficient precision of prediction models and help load balancing on one hand and lower the communication line load on the other hand?

- How to efficiently identify dependent information for producing complex measurements across more elements in the grid?

- What is the acceptable age of particular type of information for a given stakeholder?

\section{G. Evaluation of Smart Grid communication strategies}

Modelling of different communication strategies in the Smart Grid is essential for the evaluation of grid behavior in different situations. Identification of a suitable communication strategy is often hindered with a set of constraints that must be fulfilled. For instance, one of the primary restrictions in the Czech Republic is the employment of existing communication infrastructure, because significant rebuild of the infrastructure would unacceptably increase the Smart Grid deployment costs. When following the physical structure of the grid described above, we need to transfer measurements from Smart Meter units placed at customers electrical connections to a datacenter for processing, and control commands back to the elements of the grid. The problem is that a Smart Meter cannot be usually connected directly to the computer network to send all required data securely over the Internet.

Employment of the Internet connection of the customer for direct connection of the Smart Meter to the Internet would be a solution, but in terms of its availability, reliability and the legal complexity, it is merely impossible to deploy it nationwide.

There are two available groups of technologies that can be used in Smart Grids to transfer data between the elements of the grid-data transfer over power lines and wireless data transfer. Possible use and issues associated with these technologies in the Czech Smart Grid are examined in Section IV.

Relevant research questions for this area are:

- Can network modeling and network simulation tools be used in large scale as Smart Grid modeling and validation requires? What optimizations need to be done prior their use?

- What level of precision in a network simulation is required for trustworthy validation of the communication strategy?

- What communication channels and communication strategies are suitable for control channel? What are the requirements that control channels need to meet?

- Is it better to transfer raw measurements or pre-processed data? Where does the processing take place?

- How can we increase the reliability of the line? How can we get cost-effective line redundancy?

\section{H. Quality assessment of different Smart Grid design options}

The quality evaluation, prediction and optimization during Smart Grid design are critical cross-cutting concerns to all the research issues discussed above. The Smart Grid quality is typically understood in terms of the grid reliability, security and efficiency, and considered along with its environmental and energy sustainability and cost.

The reliability engineering in the Smart Grid domain aims at incorporating autonomous control actions to enhance reliability by increasing resiliency against component failures and natural disasters, and by minimizing frequency and magnitude of power outages subject to regulatory policies, operating requirements, equipment limitations, and customer preferences [23]. This includes the techniques for automated failure detection, isolation and restoration (FDIR), and terms such as grid survivability and robustness.

The security requirements for a Smart Grid are mainly the authenticity, integrity, and privacy of metering data, along with the safety and availability of the grid [24]. The Smart Grid design shall aim at resiliency against malicious attacks through better physical security and cybersecurity, to protect the infrastructure, energy asset and data of the grid.

The efficiency of a Smart Grid refers to the infrastructure utilization on both the energy-transfer and data-transfer level. The new research questions emerge mainly in the context of data-transfer efficiency, which is specifically concerned with 
the level of utilization of communication channels and latency of data delivery.

The research questions concerned with the quality assessment and optimization include:

- Which of two or more Smart Grid design options is better with respect to a specific quality attribute?

- How shall a specific Smart Grid design alternative be changed to optimize a specific quality attribute (under given constraints, such as the cost)? E.g. to optimize the time needed for failure detection, isolation and grid restoration.

- What is the quality trade-off of different quality-related strategies? E.g. what is the security improvement and efficiency degradation of a specific security strategy?

- What is the context (characteristics of the settings) in which a specific quality-improving strategy yields highest benefits?

- Can the Smart Grid reach a specific unsafe condition (i.e. a state violating safety specification)?

- What are the critical components of the Smart Grid with respect to a specific quality attribute? E.g. what are the most critical components with respect to grid security or survivability? What communication links have the highest impact on data-transfer latency?

- What is the level of satisfaction of individual stakeholders, including the energy distributors, customers and public organizations?

Following on from these research questions the next section describes in detail various communication strategies that are being currently evaluated by Czech power distribution companies for potential deployment in their Smart Grid projects.

\section{SitUATIONAL STUDY OF THE COMMUNICATION STRATEGY FOR THE CZECH SMART GRID}

Together with our industrial partner, the Mycroft Mind company, which is a leading expert in the field of Smart Grid simulation in the Czech Republic, we are researching new ways of Smart Grid modelling, simulation and analysis that can be integrated into their analytical tool called Grid Mind.

One of the biggest challenges in Smart Grid deployment in the Czech Republic is the communication strategy. The Advanced Metering Infrastructure (AMI) produces waste amount of data that needs to be transferred to a datacenter of the power distributor for processing. Every day the grid of the CEZ power distributor is expected to produce more than 340 Million measurements. The key concern is that the current communication channels will not be able to transfer the measurements in time. Another challenge is to process the measured data at real time, which is necessary for effective demand response optimization. Last, there is a concern that the control channels will not be able to deliver the control commands with the necessary level of reliability.

Our colleagues at the Faculty of Informatics, Masaryk University, Brno, were involved in the research examining what transfer characteristics can be expected with different versions of GPRS. We now look at this problem in a broader way. Together with Mycroft Mind we are building a modelling and simulation environment that is planned to be used for modelling, simulation and validation of various communication strategies in the Smart Grid, whose key characteristics are elaborated in the rest of this section.

There are two available groups of technologies that can be used in a Smart Grid to transfer data between the elements of the grid-data transfer over power lines and wireless data transfer.

The Power Line Carrier (PLC) technology stands for a family of communication protocols and technologies that transfer data over power lines. Different PLC technologies vary in frequency ranges used as transport medium for data NPL (Narrow Power Line) uses frequencies between $3 \mathrm{KHz}$ and $148.5 \mathrm{KHz}, \mathrm{BPL}$ (Broadband Power Line) uses higher frequency range between $2 \mathrm{MHz}$ and $50 \mathrm{MHz}$. The quality of the data transfer is significantly dependent on the properties of the power line, such as the path attenuation and noise in case of medium voltage lines connecting primary to secondary substations. The number of parameters that need to be evaluated increases when low voltage lines that connect secondary substations and Smart Meter devices are used. More detailed characteristics of PLC technologies can be found in [25].

In the low voltage network there are two types of devices: central low voltage unit (CLU) and remote low voltage unit (RLU). CLU is located at low voltage substations usually with a few hundreds of connected customers. RLU is built into a Smart Meter and may act also as a repeater in order to extend the action radius of the PLC signal. The PLC communication medium is composed of sub networks defined by CLU. To avoid collisions, access to PLC medium has to be controlled, therefore CLU controls all the traffic and RLU are allowed to communicate only in case they respond to CLU's request

PLC was chosen as a suitable technology for centralized collection of measurements from Smart Meters. The benefit is that it uses existing power lines which decreases deployment costs and provides sufficient communication channel for collection of power consumption measurements. The deployment of this technology is currently being validated using almost 40,000 Smart Meter units deployed in four Czech towns [26]. The problem is how to transfer the collected data from substations.

The latter group of technologies usable for data transfers in the Smart Grid is wireless transfer. There are many available wireless communication technologies that can be effectively used for measurements transfer to a data center for processing. We discuss some of the technologies usable over large distance and explain their advantages and disadvantages below.

a) $2 G$ GPRS/EDGE: In the Czech Republic, the coverage of public cellular networks is approaching $99 \%$ of area. $2 \mathrm{G}$ cellular networks provide data services in the speed of kbps or tens kbps. These data services are optimally tailored for automated meter readings. Mobile operators offer dataonly SIM cards for industrial use with lower communication priority. No investments into network infrastructure are needed 
for cellular network-based AMI solutions. Transferring data over 2G cellular network from each individual Smart Meter unit would not be cost effective in terms of running costs paid to mobile operator, therefore we propose to use this technology to transfer collected data from substation. It is necessary to model such a situation and validate if the transfer speed of $2 \mathrm{G}$ networks is sufficient.

b) $3 G$ UMTS: $3 \mathrm{G}$ cellular networks are extending the functionality of traditional $2 \mathrm{G}$ networks, allowing data transfers at significantly higher speeds. With HSPA extension peak data rates up to $14 \mathrm{Mbit} / \mathrm{s}$ in the downlink and $5.75 \mathrm{Mbit} / \mathrm{s}$ in the uplink are supported. Data rates of $3 \mathrm{G}$ networks are fully sufficient for AMI purposes. In the Czech Republic, most of the $3 \mathrm{G}$ coverage is in densely populated areas. According to the data provided by the Czech Telecommunication Office, the mobile operator with the best $3 \mathrm{G}$ signal coverage covered at the end of year 2012 only $48 \%$ of the Czech Republic [27]. Substations in uncovered areas will have to rely on slower $2 \mathrm{G}$ networks.

c) 4G LTE: LTE cellular network rapidly increases transfer speeds up to $100 \mathrm{Mbit} / \mathrm{s}$. Mobile operators started to deploy this communication technology in the Czech Republic at the end of 2013. The Vodafone mobile operator selected this technology as a complement to their $3 \mathrm{G}$ network which will not be extended anymore. They would like to primarily upgrade 2G EDGE connection in places uncovered by HSPA to cover $93 \%$ of the Czech population with $3 \mathrm{G}$ or $4 \mathrm{G}$ connectivity until December 2014. Such a rapid deployment of LTE as a replacement of $2 \mathrm{G}$ network should be considered in evaluation of Smart Grid communication model in case of $2 \mathrm{G}$ networks insufficient speed.

d) WLAN/WiFi: Broadband wireless technologies based on the IEEE 802.11 standard have found broad acceptance worldwide for wireless area networking. The use of WLANs for AMR has been frequently reported. In the US, several cities (e.g. Santa Clara, CA) already use WLANs to automatically collect readings from electricity, gas, and water meters [28]. Using $802.11 \mathrm{~s}$ technology supporting mesh may provide a technically attractive solution for AMR [28]. Use of public WLAN in municipalities, if available, may solve problems with connection availability at substations, but considerable number of substations is out of reach of WLANs. Using WLAN as primary connection technology for measurements collection is less reliable than PLC.

e) WiMAX: Worldwide Inter-operability for Microwave Access is a wireless broadband access technology which has been designed to provide transfer speeds up to $72 \mathrm{Mbit} / \mathrm{s}$ in both directions. Communication range varies between few kilometers in case of non-line of sight conditions and $50 \mathrm{~km}$ in case of line of sight conditions. Deployment of a dedicated WiMAX network solely for the purpose of an AMI likely cannot be justified. In those areas where public WiMAX services are available (e.g. municipal networks), WiMAX may principally be an option [28]. f) ZigBee: ZigBee standards-based protocols provide infrastructure for wireless sensor network applications. ZigBee is capable of creating a mesh network, which provides additional capabilities-increased redundancy, self-configuration and self-healing. Situation of building nationwide dedicated network using ZigBee is the same like in case of WiMAX. Possible use of ZigBee wireless network in terms of Smart Metering is connection of individual sensors where the power line does not meet quality requirements of PLC based protocol. Another use may be peer-to-peer connection between substations to increase redundancy or capacity of Internet connection (e.g. EDGE).

g) Satellite systems: Satellite system are suitable for sending measurements in areas where no suitable terrestrial infrastructure is available. Today, there is a number of satellite operators offering data transfer services. For instance Iridium service, which uses low earth orbit satellites or SpaceChecker that uses geostationary satellites. Deployment in areas covered by other communication infrastructures needs to be evaluated, because costs are expected to be significantly higher than use of other terrestrial communication technologies.

The infrastructure that is currently examined in the Czech Republic combines PLC technology to transfer measurements from Smart Meters to a substation. Each substation is collecting data usually from a few hundreds Smart Meters. The data concentrator unit, which is deployed at substations, can process data locally which opens new possibilities in building more decentralized solutions. Each data concentrator is equipped with a cellular network modem, in the Czech Republic usually EDGE technology, and sends data to a data center. This approach brings significant improvement in terms of connection costs reduction but increases the demands on speed and reliability of Internet connection available at substations, because more collected data needs to be transferred from a single point.

We were provided with the operational measurements of data transfer speed and latency of currently used GPRS communication channel in the test deployment of Smart Grid in the Czech Republic. The used low-priority industrial SIM cards can utilize only $10 \%$ of the communication capacity of a mobile base transceiver station (BTS), therefore under heavy load they operate only at the speed of few Kbits per second. Provided measurements show problems with connection handling across different levels of the network stack. If there is a network connection that is not used for a longer period of time, this connection is disconnected by the BTS at the physical level, but the TCP connection remains open. Any later attempt to transfer data via this broken TCP connection results in a communication failure and a need to reopen the connection which takes a few seconds to establish. The discussed problems illustrate the need to build a valid simulation model of the network communication in the Smart Grid. To be able to handle the high complexity of this model, we propose together with our industrial partner Mycroft Mind decomposition of this model into three interconnected simulation models. The 
first model describes the simplified behavior of the physical layer, the second model describes the simplified version of the transport protocol and the third model describes used application protocols. The Grid Mind simulation environment utilizes the discrete event simulation and proposed models to evaluate different communication channels, protocols and communication strategies.

Constraint in a form of available Internet connection at the locality of the substation is one of the key problems that needs to be evaluated using correct modelling and simulation approaches.

\section{Conclusions}

To minimize the risks associated with the Smart Grid deployment and maximize its quality across multiple quality attributes, possible modelling and simulation techniques to evaluate and compare different Smart Grid design alternatives before the actual deployment are becoming of high interest. In this paper, we elaborated a discussion on the possible directions of Smart Grid modelling and analysis to reach the "quality by design", and presented specific research questions towards Smart Grid design that we identified with our industrial partners. One of the research questions, concerned with the communication strategy, was then discussed in detail and linked to the specific situation in the Czech Republic. The communication strategy evaluation is at the same time our ongoing research aim, which will be in the future extended in the direction of the research questions discussed in this paper.

\section{ACKNOWLEDGEMENT}

We would like to thank our industrial partners, especially the Mycroft Mind company and its head Filip Prochazka, for the fruitful discussions and valuable inputs that made this paper possible.

\section{REFERENCES}

[1] J. Bremer and M. Sonnenschein, "A distributed greedy algorithm for constraint-based scheduling of energy resources," in Proc. of FedC SIS'12. IEEE, 2012. ISBN 978-1-4673-0708-6 pp. 109-115.

[2] Y. Simmhan, S. Aman, B. Cao, M. Giakkoupis, A. Kumbhare, Q. Zhou, D. Paul, C. Fern, A. Sharma, and V. Prasanna, "An informatics approach to demand response optimization in smart grids," Computer Science Department, University of Southern California, Tech. Rep., 2011.

[3] EU, "Standardization mandate to european standardisation organisations (ESOs) to support european smart grid deployment," March 2011, las retrieved 2014-04-21. [Online]. Available: http://ec.europa.eu/energy/ gas_electricity/smartgrids/doc/2011_03_01_mandate_m490_en.pdf

[4] X. Fang, S. Misra, G. Xue, and D. Yang, "Smart grid - the new and improved power grid: A survey," Communications Surveys Tutorials, IEEE, vol. 14, no. 4, pp. 944-980, 2012. doi 10.1109/SURV.2011.101911.00087

[5] EU, "Single market for gas \& electricity: Smart grids," July 2013, last retrieved 2014-04-21. [Online]. Available: http://ec.europa.eu/energy/ gas_electricity/smartgrids/smartgrids_en.htm

[6] EU, "Standardisation mandate to CEN, CENELEC and ETSI in the field of measuring instruments for the development of an open architecture for utility meters involving communication protocols enabling interoperability," March 2009, last retrieved 201404-21. [Online]. Available: http://ec.europa.eu/energy/gas_electricity/ smartgrids/doc/2009 03_12_mandate_m441_en.pdf

[7] OPEN meter, July 2012, last retrieved 2014-04-21. [Online]. Available: http://www.openmeter.com/
[8] M. Alberto, R. Soriano, J. Gotz, R. Mosshammer, N. Espejo, F. Lemenager, and R. Bachiller, "OpenNode: A smart secondary substation node and its integration in a distribution grid of the future," in Proc. of FedCSIS'12. IEEE, 2012. ISBN 978-1-4673-0708-6 pp. 1277-1284.

[9] R. Hassan and G. Radman, "Survey on smart grid," in Proc. of IEEE SoutheastCon'10, 2010. doi: 10.1109/SECON.2010.5453886 pp. 210 213.

[10] W. Wang, Y. Xu, and M. Khanna, "A survey on the communication architectures in smart grid," Computer Networks, vol. 55, no. 15, pp. 3604-3629, 2011. doi: 10.1016/j.comnet.2011.07.010

[11] S. Amin and B. Wollenberg, "Toward a smart grid: power delivery for the 21st century," Power and Energy Magazine, IEEE, vol. 3, no. 5, pp. 34-41, 2005. doi: 10.1109/MPAE.2005.1507024

[12] M. Pipattanasomporn, H. Feroze, and S. Rahman, "Multi-agent systems in a distributed smart grid: Design and implementation," in Proc. of PSCE'09, 2009. doi: 10.1109/PSCE.2009.4840087 pp. 1-8.

[13] S. D. Ramchurn, P. Vytelingum, A. Rogers, and N. Jennings, "Agentbased control for decentralised demand side management in the smart grid," in Proc. of AAMAS'11. ACM, 2011, pp. 5-12.

[14] P. McDaniel and S. McLaughlin, "Security and privacy challenges in the smart grid," Security Privacy, IEEE, vol. 7, no. 3, pp. 75-77, 2009. doi: 10.1109/MSP.2009.76

[15] H. Khurana, M. Hadley, N. Lu, and D. Frincke, "Smart-grid security issues," Security Privacy, IEEE, vol. 8, no. 1, pp. 81-85, 2010. doi: 10.1109/MSP.2010.49

[16] A. Metke and R. Ekl, "Security technology for smart grid networks," IEEE Transactions on Smart Grid, vol. 1, no. 1, pp. 99-107, 2010. doi: 10.1109/TSG.2010.2046347

[17] D. S. Menasché, R. M. Meri Leäo, E. de Souza e Silva, A. Avritzer, S. Suresh, K. Trivedi, R. A. Marie, L. Happe, and A. Koziolek, "Survivability analysis of power distribution in smart grids with active and reactive power modeling," SIGMETRICS Perform. Eval. Rev., vol. 40, no. 3, pp. 53-57, Jan. 2012. doi: 10.1145/2425248.2425260

[18] A. Conejo, J. Morales, and L. Baringo, "Real-time demand response model," Smart Grid, IEEE Transactions on, vol. 1, no. 3, pp. 236-242, 2010. doi: 10.1109/TSG.2010.2078843

[19] CEPS, "Schéma rozvodné sítě v ČR (schema of Czech transmission network)," 2013, last retrieved 2014-04-21. [Online]. Available: http://www.ceps.cz/CZE/Cinnosti/Technicka-infrastruktura/ PublishingImages/Mapa siti_CZ.PNG

[20] EU, "Communication from the commision to the european council and the european parlament - an energy policy for europe," January 2007, last retrieved 2014-04-21. [Online]. Available: https: //www.europia.eu/Content/Default.asp?PageID=412\&DocID=13922

[21] EPRI, "Electricity energy storage technology options," December 2010 last retrieved 2014-04-21. [Online]. Available: http://www.epri.com/ abstracts/Pages/ProductAbstract.aspx?ProductId=000000000001020676

[22] P. Vytelingum, T. D. Voice, S. D. Ramchurn, A. Rogers, and N. R Jennings, "Agent-based micro-storage management for the smart grid," in Proc. of AAMAS '10, 2010, pp. 39-46.

[23] K. Moslehi and R. Kumar, "A reliability perspective of the smart grid," IEEE Trans. on Smart Grid, vol. 1, no. 1, pp. 57-64, June 2010. doi: 10.1109/TSG.2010.2046346

[24] D. von Oheimb, "It security architecture approaches for smart metering and smart grid," in Proc. of SmartGridSec'12, ser. LNCS, vol. 7823. Springer, 2013. doi: 10.1007/978-3-642-38030-3_1 pp. 1-25.

[25] OPEN meter, "Description of current state-of-the-art of technology and protocols - description of state-of-the-art plc-based access technology," May 2009, last retrieved 2014-04-21. [Online] Available: http://www.openmeter.com/files/deliverables/OPEN-Meter $\% 20 \mathrm{WP} 2 \backslash \% 20 \mathrm{D} 2.1 \backslash \% 20$ part2 $\backslash \% 20 \mathrm{v} 2.3$.pdf

[26] CEZ, "Čez smart grids inteligentní měření,” January 2014, last retrieved 2014-04-21. [Online]. Available: http://www.futuremotion.cz/ smartgrids/cs/index.html

[27] CTU, "Výroční zpráva českého telekomunikačního úřadu za rok 2012 (annual report of the czech telecommunication office for 2012)," April 2013, last retrieved 2014-04-21. [Online]. Available: http://www.ctu.cz/ cs/download/vyrocni zpravy/vyrocni zprava_ctu_2012.pdf

[28] OPEN meter, "Description of current state-of-the-art technologies and protocols - general overview of state-of-the-art technological alternatives," June 2009, last retrieved 2014-04-21. [Online]. Available: http://www.openmeter.com/files/deliverables/OPEN-Meter $\% 20 \mathrm{WP} 2 \backslash \% 20 \mathrm{D} 2.1 \backslash \% 20$ part $\backslash \% 20 \mathrm{v} 3.0 . \mathrm{pdf}$ 